\title{
Foxo1 mediates insulin action on apoC-III and triglyceride metabolism
}

\author{
Jennifer Altomonte, ${ }^{1}$ Lin Cong, ${ }^{1}$ Sonal Harbaran, ${ }^{1}$ Anja Richter, ${ }^{1}$ Jing Xu, ${ }^{1}$ \\ Marcia Meseck, ${ }^{1}$ and Hengjiang Henry Dong ${ }^{1,2}$
}

\begin{abstract}
1Department of Gene and Cell Medicine, ${ }^{2}$ Division of Experimental Diabetes and Aging, Mount Sinai School of Medicine, New York, New York, USA.
\end{abstract}
\begin{abstract}
The apolipoprotein apoC-III plays an important role in plasma triglyceride metabolism. It is predominantly produced in liver, and its hepatic expression is inhibited by insulin. To elucidate the inhibitory mechanism of insulin in apoC-III expression, we delivered forkhead box O1 (Foxo1) cDNA to hepatocytes by adenovirus-mediated gene transfer. Foxo1 stimulated hepatic apoC-III expression and correlated with the ability of Foxo1 to bind to its consensus site in the apoC-III promoter. Deletion or mutation of the Foxo1 binding site abolished insulin response and Foxo1-mediated stimulation. Likewise, Foxo1 also mediated insulin action on intestinal apoC-III expression in enterocytes. Furthermore, elevated Foxo1 production in liver augmented hepatic apoC-III expression, resulting in increased plasma triglyceride levels and impaired fat tolerance in mice. Transgenic mice expressing a constitutively active Foxo1 allele exhibited hypertriglyceridemia. Moreover, we show that hepatic Foxo1 expression becomes deregulated as a result of insulin deficiency or insulin resistance, culminating in significantly elevated Foxo1 production, along with its skewed nuclear distribution, in livers of diabetic NOD or $d b / d b$ mice. While loss of insulin response is associated with unrestrained apoC-III production and impaired triglyceride metabolism, these data suggest that Foxo1 provides a molecular link between insulin deficiency or resistance and aberrant apoC-III production in the pathogenesis of diabetic hypertriglyceridemia.
\end{abstract}

\section{Introduction}

One of the most abundant apolipoproteins in plasma, apoC-III is present as an exchangeable moiety between HDL- and triglyceride-rich (TG-rich) particles, such as VLDL and chylomicrons. apoC-III functions as an inhibitor of lipoprotein lipase (LPL), a key enzyme in the hydrolysis of TG in VLDL and chylomicrons $(1,2)$. At higher concentrations, apoC-III also inhibits hepatic lipase activity (3). In addition, increased apoC-III content adversely affects apoE-mediated hepatic uptake of TG-rich remnants (4-6). Thus, elevated plasma apoC-III levels are associated with impaired hydrolysis and retarded clearance of TG-rich particles, resulting in the accumulation of VLDL-TG and chylomicrons in plasma and the development of hypertriglyceridemia $(7,8)$. Conversely, apoC-III deficiency, caused by genetic disruption of the apoC-III gene, is associated with hypotriglyceridemia due to enhanced hydrolysis and clearance of TG-rich particles (9-11). Thus, apoC-III plays an important role in TG metabolism. apoC-III is mainly produced in liver, and its hepatic production is subject to insulin inhibition $(12,13)$, but the underlying molecular basis for this inhibition remains elusive.

Forkhead box O1 (Foxo1) is a nuclear transcription factor that belongs to a protein family characterized by a highly conserved DNA binding motif, termed the forkhead box. Foxo1 is a substrate of Akt/protein kinase B and serum- and glucocorticoidinducible kinase (SGK), and it plays an important role in insulin

Nonstandard abbreviations used: ChIP, chromatin immunoprecipitation; EMSA, electrophoretic mobility shift assay; Foxo1, forkhead box O1; GK, glucokinase; IRE, insulin response element; LPL, lipoprotein lipase; NON, nonobese nondiabetic; PEPCK, phosphoenolpyruvate carboxykinase; SGK, serum- and glucocorticoidinducible kinase; TG, triglyceride.

Conflict of interest: The authors have declared that no conflict of interest exists.

Citation for this article: J. Clin. Invest. 114:1493-1503 (2004).

doi:10.1172/JCI200419992. signaling (14-18). In the absence of insulin, Foxo1 resides in the nucleus, acting as a trans-activator to enhance promoter activity. In response to insulin, Foxo1 is phosphorylated in a PI3K-dependent manner, resulting in its nuclear exclusion. There are several highly conserved phosphorylation sites in Foxo1, and mutations at these phosphorylation sites lead to failure in phosphorylation, resulting in constitutive nuclear localization and trans-activation of target gene expression (19-21).

To investigate the mechanism underlying the inhibitory effect of insulin on apoC-III expression, we studied the role of Foxo1 in hepatic apoC-III regulation. We show that Foxo1 is an effective mediator of insulin in modulating hepatic apoC-III expression, affecting plasma TG metabolism. Adenovirus-mediated Foxo1 production in hepatocytes augmented hepatic apoC-III expression, which was counteracted by insulin. Elevated Foxo1 production in liver resulted in impaired TG metabolism in mice. Transgenic mice expressing a constitutively active Foxo 1 allele exhibited hypertriglyceridemia. In diabetic NOD and $d b / d b$ mice, hepatic Foxo1 expression became deregulated, as evidenced by elevated Foxo1 production along with its increased nuclear localization in liver. These results shed light on the mechanism underlying the inhibitory effect of insulin on apoC-III expression, suggesting that Foxo1 deregulation associated with insulin deficiency or insulin resistance plays an important role in linking impaired insulin action to aberrant apoC-III production in the pathophysiology of diabetic hypertriglyceridemia.

\section{Results}

Effects of Foxo1 on hepatic apoC-III expression. Previous studies indicated that hepatic apoC-III expression is negatively regulated by insulin $(12,13)$, but the underlying mechanism is unknown. To study the molecular mechanism underlying this inhibitory effect of insulin on hepatic apoC-III expression, we examined the effect of Foxo1 

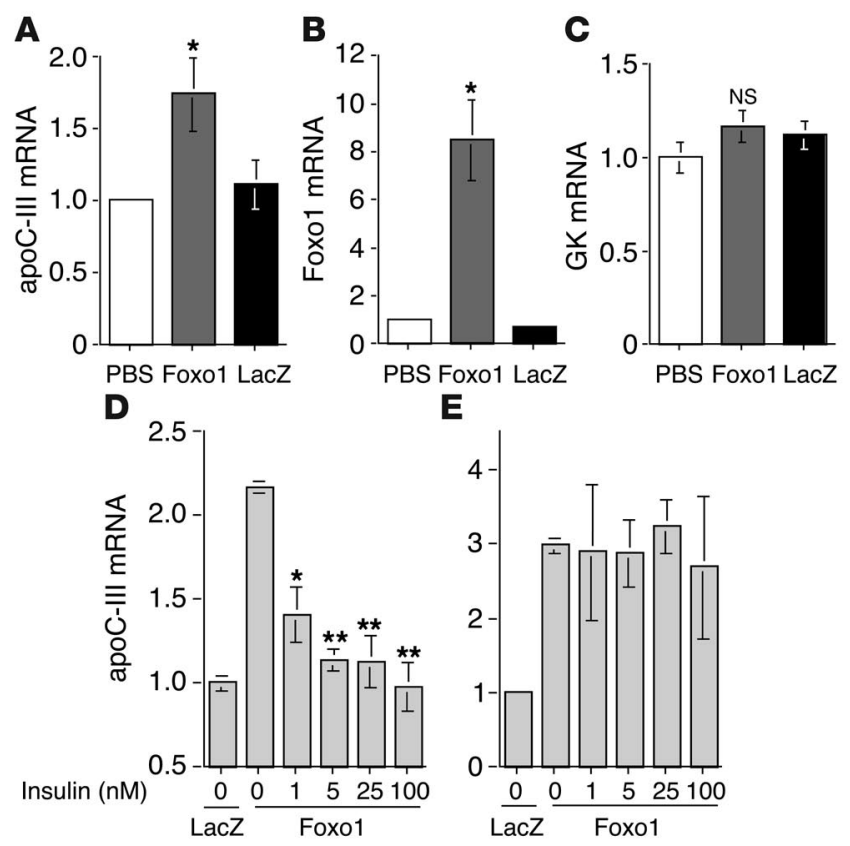

E

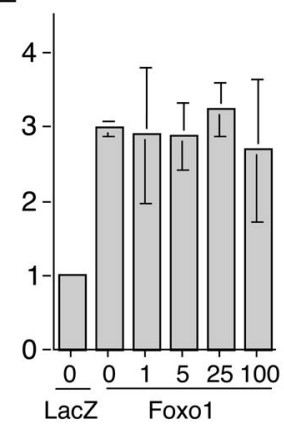

on hepatic apoC-III expression in cultured hepatocytes. Using an adenovirus-mediated gene delivery system, we transferred the wildtype Foxo 1 cDNA to cultured rat hepatocytes and determined the intracellular apoC-III mRNA levels following 24 hours of Foxo1 transgene expression. Adenovirus-mediated production of Foxo1 significantly stimulated endogenous apoC-III expression (Figure 1A), which correlated with Foxo1 expression in hepatocytes (Figure 1B). In contrast, the level of apoC-III expression in control vectortransduced hepatocytes remained unchanged. To demonstrate that elevated hepatic apoC-III production in Foxo1 vector-transduced hepatocytes is attributable to the specific effect of Foxo1, we determined the expression level of glucokinase (GK), a glycolytic enzyme that is not regulated by Foxo1 (22), following Foxo1 expression in hepatocytes. As shown in Figure 1C, Foxo1 production did not affect GK expression, as reflected by the lack of changes in the relative level of GK mRNA in Foxo1 vector- versus control vector-transduced cells. These results demonstrate that Foxo1 stimulates hepatic apoC-III expression in cultured primary hepatocytes.

To study whether Foxo1 is an effective mediator of insulin in regulating hepatic apoC-III production, we transferred Foxo1 cDNA into HepG2 cells. Unlike primary hepatocytes, HepG2 cells express little Foxo1, so that the effect of Foxo1 on hepatic apoC-III expression would have to be ascribed to exogenous Foxo1. Here, we studied the endogenous expression level of apoC-III induced by Foxo1 transduction in the presence and absence of insulin. As shown in Figure 1D, adenovirus-mediated Foxo1 production significantly stimulated apoC-III mRNA expression, but the stimulatory effect was inhibited by insulin in a concentrationdependent manner. Insulin appeared to play a dominant role in inhibiting apoC-III expression, as the inhibitory effect of insulin was detected in the presence of Foxo1. To corroborate this finding, we expressed a constitutively active Foxo1 allele, Foxo1-ADA, in HepG 2 cells. Foxo1-ADA contains 3 amino acid substitutions at three conserved phosphorylation sites and is unable to undergo insulin-dependent phosphorylation (17). As a result, Foxo1-ADA is associated with constitutive trans-activation of target gene expres-

\section{Figure 1}

Effects of Foxo1 on hepatic apoC-III expression. Rat primary hepatocytes were transduced with Foxo1 or LacZ vector at an MOI of $50 \mathrm{PFU} / \mathrm{cell}$ or mock-transduced with PBS. After 24 hours of transduction, the intracellular levels of apoC-III (A), Foxo1 (B), and GK (C) mRNA were determined by real-time RT-PCR using $\beta$-actin mRNA as control. The effect of Foxo1 on hepatic apoC-III expression in response to insulin was assayed in HepG2 cells. Cells were transduced with Foxo1, Foxo1-ADA, or control LacZ vector (50 PFU/ cell) in the absence or presence of insulin at different concentrations. Twenty-four hours after transduction, cells were collected for determination of the intracellular levels of apoC-III mRNA induced by Foxo1 (D) and Foxo1-ADA (E). ${ }^{*} P<0.05,{ }^{* *} P<0.005$; significantly different from controls. NS, not significant by ANOVA. Data were from 3 independent experiments.

sion (17). Indeed, adenovirus-mediated production of Foxo1-ADA resulted in a 3 -fold induction of hepatic apoC-III expression in HepG2 cells. Unlike its wild-type counterpart, Foxo1-ADA-mediated stimulation was no longer subject to insulin inhibition, as the elevated apoC-III mRNA levels in Foxo1-ADA vector-transduced cells remained unchanged irrespective of the addition of insulin into culture media (Figure 1E).

To elucidate the molecular mechanism of the Foxo1-mediated stimulatory effect on hepatic apoC-III expression, we constructed an apoC-III promoter-directed luciferase reporter system in plasmid pHD317, in which the firefly luciferase cDNA was directed by the human APOC3 promoter (Figure 2A). We then transfected pHD317 into HepG2 cells in the presence and absence of Foxo 1 expression using a $\beta$-gal-expressing plasmid as an internal standard for the normalization of transfection efficiency. In the absence of Foxo 1 expression, transfection with pHD317 resulted in only basal luciferase expression. However, cotransfection with pHD317 and the Foxo1-expressing plasmid pCMV5-Foxo1 resulted in a greater than 4-fold induction of luciferase activity (Figure 2B). To confirm that this stimulatory effect on luciferase reporter expression was via a Foxo1-dependent mechanism, we determined the luciferase expression level following coexpression of Foxo1 and its dominant-negative mutant Foxo1- $\Delta 256$. Foxo1- $\Delta 256$, containing only the amino DNA binding domain of Foxo1, binds to Foxo1-target promoters in a competitive manner and interferes with Foxo1 function in a dominant-negative manner $(17,22)$. Consistent with its role, Foxo1- $\Delta 256$ was shown to counteract the effect of Foxo 1 and reduce the luciferase reporter expression to basal levels (Figure $2 \mathrm{~B})$. These results suggest that Foxo 1 might act directly on the APOC3 promoter in stimulating hepatic apoC-III production.

Characterization of Foxo1 -target site in the human APOC3 promoter. To characterize the Foxo1-target site in the APOC3 promoter, we constructed different versions of the human $A P O C 3$ promoter by progressively deleting portions of its upstream region. As shown in Figure 2C, the resulting mutant $A P O C 3$ promoters were subcloned into the luciferase reporter system, and the transcriptional activity of each mutant promoter in response to Foxo1 production was tested in HepG2 cells. As shown in Figure 2D, deletions up to $-498 \mathrm{nt}$ in the APOC 3 promoter produced little effect on the responsiveness of the mutant promoters to Foxo1 production, as the transcriptional activity of the mutant promoters was induced to approximately the same level as that of their wild-type counterparts. However, further deletion up to $-403 \mathrm{nt}$ in the promoter completely abolished the 
A

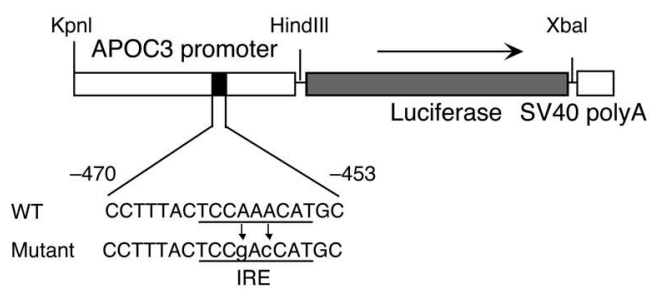

B

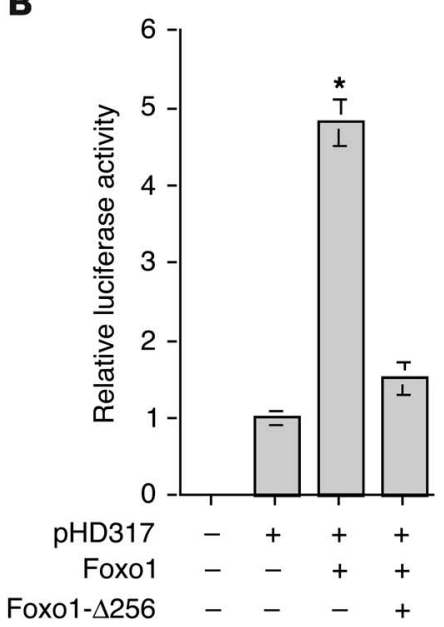

Figure 2

Effects of Foxo1 on the human APOC3 promoter activity. (A) The APOC-III promoter-directed luciferase reporter system. The wild-type and mutant IRE sequences are underlined. (B) Foxo1-mediated induction of the APOC3 promoter activity. HepG2 cells were transfected by pHD317 together with Foxo1 construct, or with both Foxo1 and Foxo1- $\Delta 256$ constructs. For each construct, $1 \mu \mathrm{g}$ of DNA for each construct was used in transfection. For normalization of transfection efficiency, $1 \mu \mathrm{g}$ pCMV5-LacZ DNA was included for normalization of transfection efficiency. (C) The APOC3 promoter variants in the luciferase reporter system. (D) Responses of APOC3 promoter variants to Foxo1 production. HepG2 cells were transfected with individual test plasmids in the absence $(-)$ or presence $(+)$ of pCMV5-Foxo1. The relative luciferase activity, after normalizing to $\beta$-gal activity, was compared between basal $(-)$ and Foxo1-inducible $(+)$ conditions. (E) Responses of wild-type and mutant APOC3 promoters to insulin. Test plasmids were transduced into HepG2 cells in the presence and absence of pCMV5-Foxo1 transfection in culture media, either supplemented with or without insulin $(30 \mathrm{nM})$. The relative luciferase activity in transduced cells was determined using $\beta$-gal activity as control. ${ }^{*} P<0.001$ vs. controls.

responsiveness of the mutant promoter to Foxo1 induction, as evidenced by the lack of changes in the transcriptional activity of the mutant promoter in the presence and absence of Foxo1 production in cells. Thus, the Foxo1 target site was confined within a small nucleotide region between -498 and -403 in the human APOC3 promoter. Consistent with this observation, this DNA region contains a putative insulin response element (IRE). To confirm this finding, we generated 2 mutant promoter variants, one of which contained a small deletion $(-498$ to -403$)$ and the other harbored 2 nucleotide substitutions (-A458C and -A460G) within the IRE DNA. The resulting mutant promoters were tested in HepG2 cells for their abilities to respond to Foxo1 induction using the luciferase reporter assay system. As shown in Figure 2D, both deletion and alterations of the IRE DNA sequence abrogated the responsiveness of the mutant promoters to Foxo1, as the transcriptional activity of the APOC 3 promoter variants remained unchanged in the presence and absence of Foxo1 production in cells.

To address whether the resulting promoter variants are associated with the loss of insulin responsiveness, we transfected pHD322 and pHD334 together with the Foxo1-expressing plasmid pCMV5-Foxo1 to HepG2 cells in the presence and absence of insulin in culture media, followed by the determination of luciferase activity in the cells after 24 hours of incubation. As shown in Figure 2E, the intracellular level of luciferase activity remained at basal levels regardless of the presence or absence of insulin. As a positive control, cotransfection of HepG2 cells with both pHD317 and pCMV5-Foxo1 plasmids resulted in about a 4-fold induction of luciferase activity, which was suppressed to a basal level by the addition of insulin into culture media. 
A

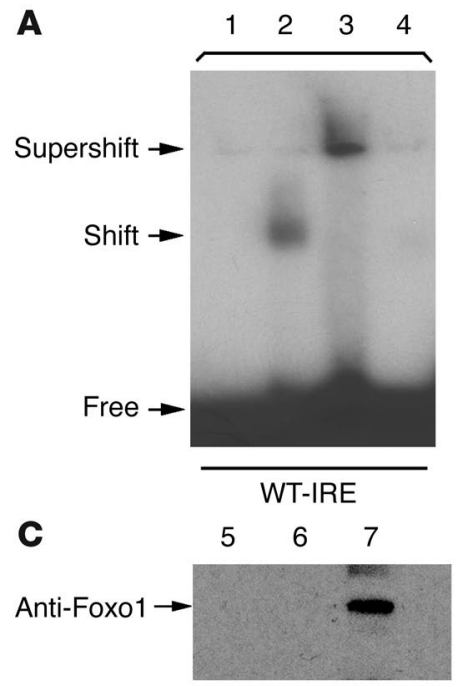

B

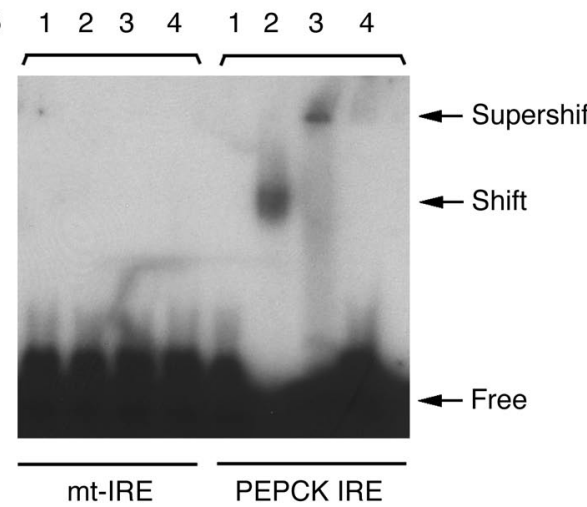

D

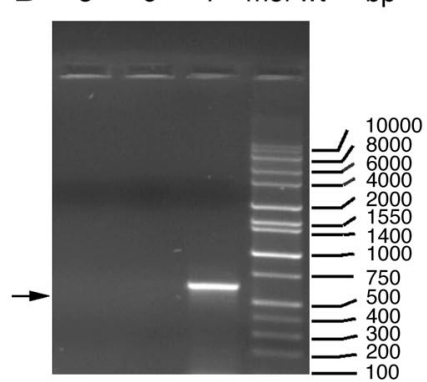

Figure 3

Molecular interaction between Foxo1 and the APOC3 promoter. Molecular association between Foxo1 and the APOC3 promoter was analyzed by EMSA and ChIP. Aliquots of Foxo1 protein from linked in vitro transcription-translation products $(5 \mu \mathrm{g})$ were incubated with $2.5 \mu \mathrm{l}$ of radioactively labeled DNA corresponding to $-467 /-440$ nt in the human APOC3 promoter (WT-IRE) (A), a mutant APOC3 IRE (mt-IRE) containing 2 substitutions, of $-\mathrm{A} 458 \mathrm{C}$ and $-\mathrm{A} 460 \mathrm{G}$, and a control PEPCK IRE DNA (B), followed by electrophoresis through $8 \%$ nondenaturing polyacrylamide gels for 30 minutes. Lane 1, DNA probe alone. Lane 2, DNA probe + Foxo1 protein lysates. Lane 3, DNA probe + Foxo1 protein lysates + anti-Foxo1 antibody $(1 \mu \mathrm{g})$. Lane 4 , DNA probe + Foxo1 protein lysates + nonlabeled competitor DNA at a molar concentration of 50 -fold excess. Free, shifted, and supershifted DNA bands were visualized by autoradiography. For ChIP assay, HepG2 cells were transduced with Foxo1 vector at an MOI of 50 PFU/cell. Cells were harvested 24 hours later and subjected to ChIP using PBS as a negative control (lane 5), control IgG (lane 6), and anti-Foxo1 antibody (lane 7). The coimmunoprecipitated chromatin DNA was analyzed by immunoblot (C) using anti-Foxo1 antibody and PCR (D) using the primers that correspond to $-655 /-20$ nt of the APOC3 promoter.

To study the molecular interaction between Foxo1 and the APOC3 promoter, we produced Foxo1 protein from a coupled in vitro transcription-translation system using pHD334, in which Foxo 1 cDNA is under the control of the T7 promoter. After verification of Foxo1 by immunoblot analysis using anti-Foxo 1 antibody, aliquots of the translation products were applied in an electrophoretic mobility shift assay (EMSA) using a radioactively labeled DNA probe from the APOC3 IRE (27 bp). As shown in Figure 3A, migration of this DNA fragment was retarded in the presence of Foxo1, resulting in a shifted DNA band. Inclusion of anti-Foxo1 antibody in the reaction mixture resulted in a supershifted DNA band, suggesting that this shifted DNA band is specific for Foxo1. In response to the addition of $50 \mathrm{M}$ excess of nonlabeled cognate DNA to the reaction mixture, both DNA retardation and supershift were abolished. As a positive control, a previously characterized IRE DNA (28 bp) from phosphoenolpyruvate carboxykinase (PEPCK) promoter was applied to EMSA (23), and similar results were produced (Figure $3 \mathrm{~B})$. As a negative control, the mutant APOC3 IRE containing 2 nucleotide substitutions (Figure 2A) was applied to EMSA and no DNA retardation was detected in the presence of Foxo1, correlating with the inability of the mutant APOC3 promoter to respond to Foxo1 stimulation (Figure 2D). Using a similar assay, we and others have previously shown that Foxo 1 is capable of binding to DNA fragments corresponding to the IRE sequences that are present in the promoter of Foxo1-targeted genes, including insulin-like growth factor binding protein 1 (IGFBP-1), PEPCK, and glucose-6-phosphatase (G6Pase) (18, 22, 24, 25).

To correlate this finding with the ability of Foxo 1 to bind the APOC 3 promoter in living cells, we performed chromatin immunoprecipitation (ChIP) on Foxo1-expressing HepG 2 cells. The rationale is that if Foxo1 binds to the APOC3 promoter, then the resulting interaction between Foxo 1 and the APOC 3 promoter DNA should be detectable through immunoprecipitation of the DNA-protein complex using anti-Foxo1 antibody. HepG2 cells transduced with Foxo1 vector were divided into 3 aliquots and, which were subjected to ChIP analysis using rabbit anti-Foxo1 antibody, control IgG, or PBS buffer (as a negative control), respectively. The resulting immunoprecipitates were studied by immunoblot and PCR analyses. As expected, Foxo1 was detected only in the products that were immunoprecipitated with anti-Foxo1 antibody, not with control IgG or PBS (Figure 3C). Furthermore, a small DNA fragment (676 bp) corresponding to the proximal region $(-675 /+1)$ of the APOC3 promoter was detected in the immunoprecipitates by anti-Foxo1 antibody, as analyzed by PCR (Figure $3 \mathrm{D}$ ). In contrast, no DNA products were amplified in control immunoprecipitates by the same PCR assay. These results demonstrate a direct protein-DNA interaction between Foxo1 and the APOC 3 promoter in cells.

Effects of Foxo1 on apoC-III and TG metabolism in vivo. To examine the effect of Foxo1 on hepatic apoC-III production and plasma TG metabolism in vivo, we transferred Foxo 1 cDNA into liver of mice. CD-1 mice at 12 weeks of age were stratified by body weight and randomly assigned to 1 of 3 groups to receive intravenous injections of $1.5 \times 10^{11} \mathrm{PFU} / \mathrm{kg}$ of Foxo1 vector, LacZ vector, or vector buffer, respectively, as described (22). This approach has been shown to result in transduction of hepatocytes predominantly in liver, with little transduction in extrahepatic tissues (26). Animals treated with Foxo1 vector exhibited significantly elevated fasting plasma TG levels 2 days after vector administration (Figure 4A). In contrast, fasting plasma TG levels in control vector-treated animals remained unchanged. To assess their relative abilities to tolerate fat, we challenged the mice with an oral bolus of olive oil, followed by the determination of plasma TG profiles. As shown in Figure 4B, plasma TG concentrations in the Foxo1 group were markedly elevated and remained at a significantly higher level even after 3 hours of olive oil administration $(380 \pm 25 \mathrm{mg} / \mathrm{dl}$ vs. $110 \pm 8 \mathrm{mg} / \mathrm{dl}$ in mock-treated 
A
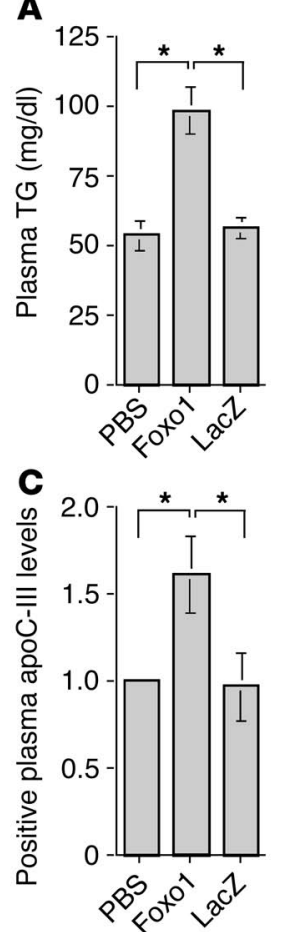

D
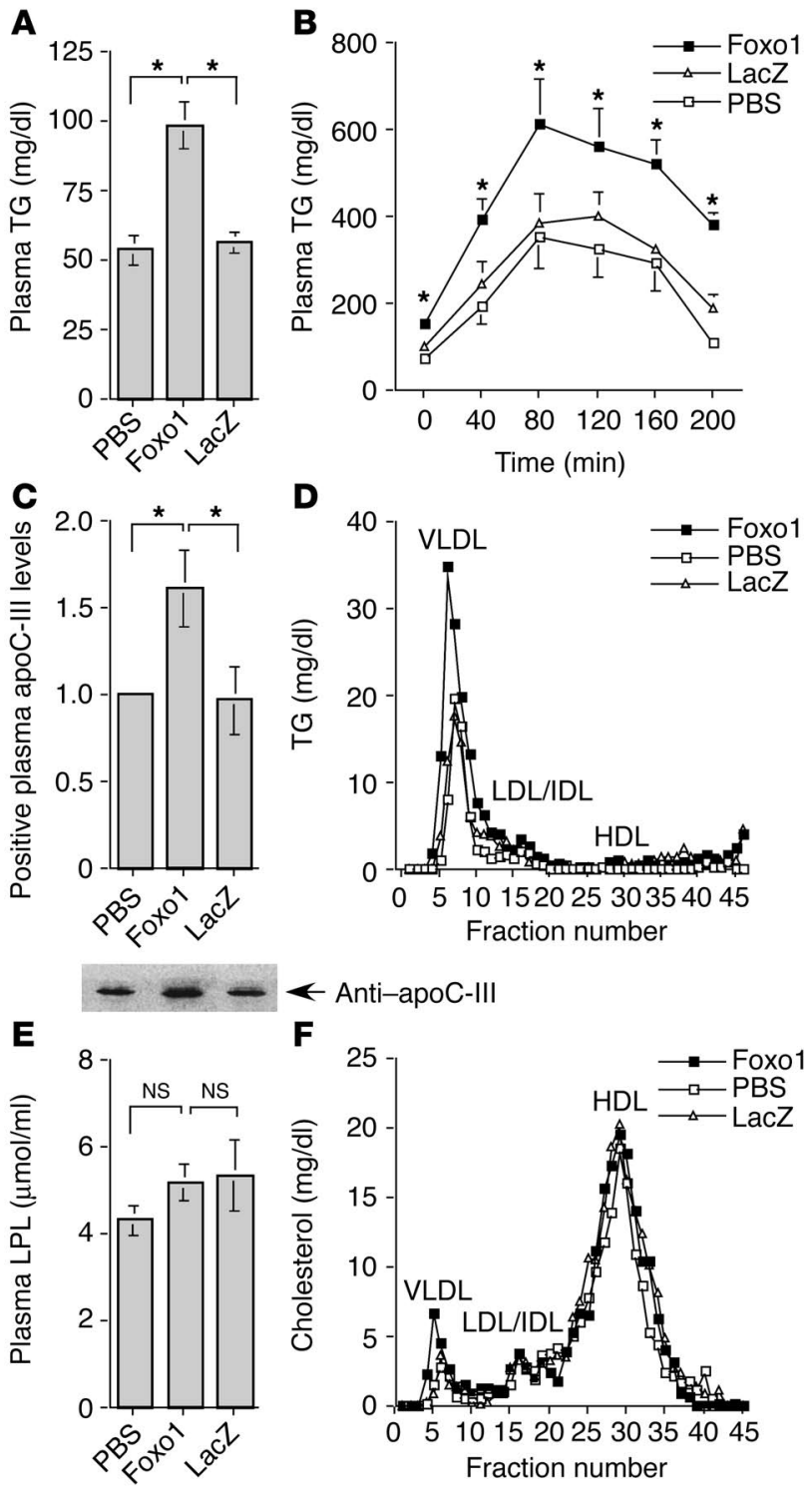

control mice; $P<0.05$ by ANOVA). In contrast, plasma TG excursion in control vector-treated animals was not significantly different from that in mock-treated controls. In addition, we determined the relative levels of plasma apoC-III by semi-quantitative immunoblot analysis. Consistent with elevated plasma TG levels in Foxo1 vector-treated mice, elevated Foxo1 production was associated with significantly increased plasma apoC-III levels (Figure 4C).

The effect of hepatic Foxo1 production on plasma TG metabolism was further illustrated following fractionation of plasma lipoproteins by gel filtration chromatography. As shown in Figure 4D, in accordance with their elevated fasting plasma TG levels, Foxo1 vector-treated mice displayed significantly increased VLDL-TG profile compared with control mice. However, the fractional concentrations of TG and cholesterol in HDL and LDL/IDL peaks in Foxo1 vector-treated mice were not significantly different from those of controls (Figure 4F). To study the effect of hepatic Foxo1 production on plasma LPL activity, we heparinized the animals by intravenous injection of heparin and took sample aliquots of tail vein blood to test the level of LPL activity. As shown in Fig-

\section{Figure 4}

Effect of Foxo1 on hepatic apoC-III and plasma TG metabolism in vivo. CD-1 mice (12 weeks old) were stratified by body weight to ensure a similar mean body weight per group $(31 \pm 1.4 \mathrm{~g}, n=6)$. The groups were Foxo1 vector-treated, LacZ vector-treated, or mock-treated. (A) Fasting plasma TG levels. Fasting plasma TG levels were determined on day 3 of hepatic Foxo1 production following an overnight fast. (B) Fat tolerance test. Plasma TG profiles in response to an oral bolus of olive oil were determined on day 4 after vector administration. (C) Plasma apoC-III levels. Mice were sacrificed after 1 week of hepatic Foxo1 production. Blood samples were collected for determination of the relative plasma apoC-III levels using a semi-quantitative immunoblot assay. A typical immunoblot is shown at the bottom of the panel. (D) TG levels in VLDL, LDL/IDL, and HDL fractions. Plasma $(400 \mu \mathrm{l})$ pooled from individual mice at day 7 after vector administration was subjected to gel filtration column chromatography. Fifty fractions $(200 \mu \mathrm{l} \mathrm{per}$ fraction) were eluted for determination of $T G$ and cholesterol levels. (E) Plasma LPL activity. Post-heparin sera were obtained from individual mice on day 5 after vector administration and used for the determination of plasma LPL activity. (F) Cholesterol levels in VLDL, LDL/IDL, and HDL fractions, as described in $\mathbf{D}$. ${ }^{*} P<0.05$ by ANOVA.

ure 4E, no significant differences were detected among different groups of animals, indicating that the observed increase in plasma VLDL-TG levels in Foxo1 vector-treated mice was not due to reduced systemic LPL levels. When total plasma cholesterol levels were determined, relatively higher cholesterol levels were detected in Foxo1 vector-treated-treated mice. However, the differences in total plasma cholesterol levels between Foxo1 vector-treated $(201 \pm 14 \mathrm{mg} / \mathrm{dl})$ and control mice $(178 \pm 22 \mathrm{mg} / \mathrm{dl}$ in mock-treated or $199 \pm 28 \mathrm{mg} / \mathrm{dl}$ in LacZ vector-treated group) did not reach statistical significance $(P>0.05$ by ANOVA).

To correlate the changes in plasma TG metabolism with the alterations in hepatic apoC-III production, we sacrificed animals after 1 week of hepatic Foxo1 production and determined the relative Foxo 1 and apoC-III mRNA levels by real-time RT-PCR using $\beta$-actin mRNA as a control. As shown in Figure 5A, hepatic apoC-III mRNA expression was significantly increased in response to Foxo 1 production in liver, which correlated with increased plasma apoC-III levels (Figure 4C) and elevated hepatic Foxo1 expression in Foxo1 vector-treated mice (Figure 5B). In addition, we studied the expression of apoA-1 and apoA-IV in response to hepatic Foxo1 production. These 2 apolipoprotein genes are clustered along with the apoC-III gene at the same locus on chromosome 11q23 (27). apoA-1 is mainly produced in liver and present as an exchangeable moiety of HDL and TG-rich particles, whereas apoA-IV is predominantly expressed in intestine and to a lesser extent in liver (28). In response to hepatic Foxo1 production, hepatic apoA-1 expression was reduced (Figure 5C), whereas the expression level of apoA-IV remained unchanged (Figure 5D). Whether this observed reduction of hepatic apoA-I expression is due to the effect of Foxo 1 or secondary to altered TG metabolism remains to be determined. In addition, we determined the body weight of mice before and 1 week after hepatic Foxo 1 cDNA delivery. No significant differences in body weight changes were detected among different groups of mice.

Plasma TG metabolism in Foxo $1^{\text {S253A }}$ transgenic mice. Foxo ${ }^{\text {S253A }}$ transgenic mice bear a constitutive Foxo1 mutant allele under the control of the transthyretin promoter and express Foxo1 ${ }^{\mathrm{S} 253 \mathrm{~A}}$ mainly in liver (29). To study the effect of hepatic Foxo $1^{\mathrm{S} 253 \mathrm{~A}}$ transgene expression on TG metabolism, we determined plasma TG levels in Foxo1 ${ }^{\mathrm{S} 253 \mathrm{~A}}$ transgenic mice. Compared with wild-type control littermates, 

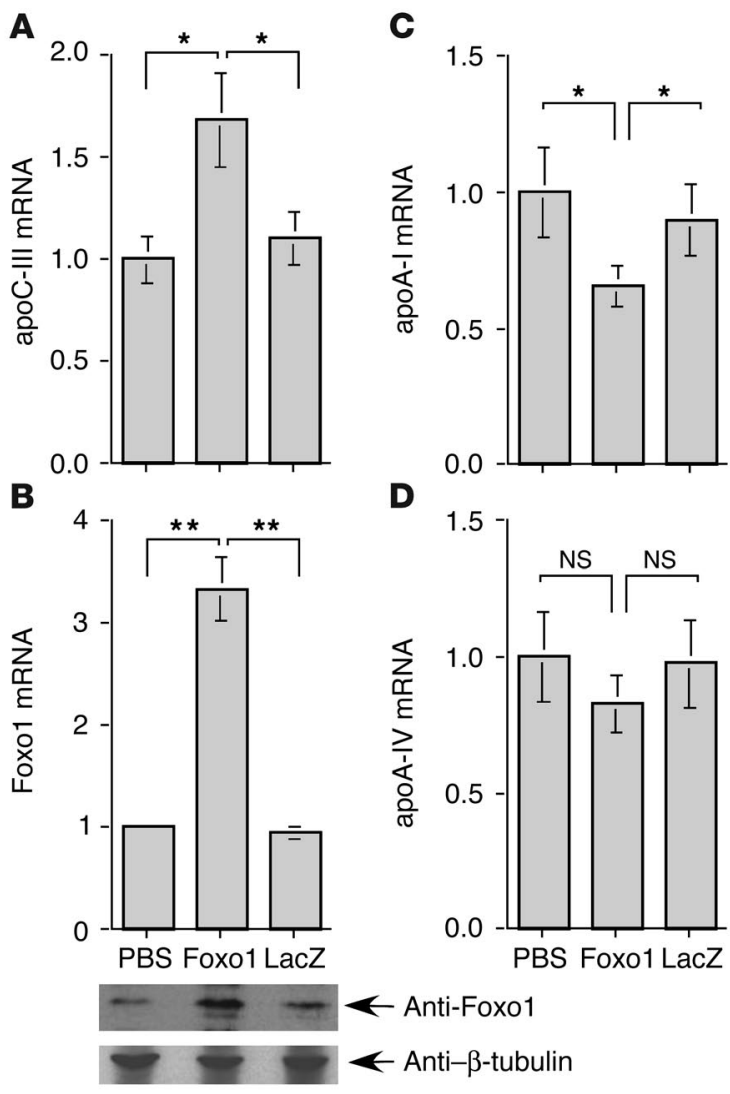

Foxo $1^{\text {S253A }}$ transgenic mice exhibited significantly elevated plasma TG levels (Figure 6A), correlating with their relatively higher plasma apoC-III levels (Figure 6B). To investigate the impact of transgenic Foxo $1^{\text {S253A }}$ expression on postprandial TG metabolism, plasma TG profiles were determined in response to an oral bolus of olive oil (Figure 6C). Plasma TG levels in Foxo $1^{\mathrm{S} 253 \mathrm{~A}}$ transgenic mice were markedly elevated $(196 \pm 28 \mathrm{mg} / \mathrm{dl}$ vs. $103 \pm 9 \mathrm{mg} / \mathrm{dl}$ in control mice, $P<0.01) 2$ hours after olive oil administration and remained at a relatively higher level $(153 \pm 38 \mathrm{mg} / \mathrm{dl}$ vs. $75 \pm 7 \mathrm{mg} / \mathrm{dl}$ in control mice, $P<0.01$ ) even after 4 hours after fat tolerance. In contrast, plasma TG levels in control littermates were only moderately raised in response to the same bolus of olive oil and returned to normal levels within 4 hours. To determine whether the impaired postprandial TG excursion was due to reduced plasma LPL activity, we subjected aliquots of tail vein blood from heparinized mice to an LPL activity assay. As shown in Figure 6D, relatively higher levels of plasma LPL activity were detected in Foxo $1^{\text {S253A }}$ transgenic mice compared with control littermates, but the difference between these 2 groups was statistically insignificant. When total plasma cholesterol levels were measured, Foxo ${ }^{\mathrm{S} 253 \mathrm{~A}}$ transgenic mice exhibited significantly higher plasma cholesterol levels $(136 \pm 7 \mathrm{mg} / \mathrm{dl}$ vs. $109 \pm 5 \mathrm{mg} / \mathrm{dl}$ in wild-type control littermates, $P<0.05$ by ANOVA).

To study the effect of Foxo 1 transgenic expression on VLDL-TG metabolism, we used gel filtration chromatography to study plasma pooled from Foxo $1^{\mathrm{S} 253 \mathrm{~A}}$ transgenic mice or control littermates. Similar to adenovirus-mediated Foxo1 production, Foxo1 transgenic expression also significantly elevated plasma VLDL-TG levels (Figure 6E). In contrast to the acute effect of adenovirus-mediated Foxo1 production on cholesterol metabolism, chronic Foxo1

\section{Figure 5}

Hepatic mRNA abundance in Foxo1 vector-treated mice. Total hepatic RNA was prepared for the determination of hepatic mRNA levels of apoC-III (A), Foxo1 (B), apoA-1 (C), and apoA-IV (D) using realtime RT-PCR. Hepatic protein extracts were prepared for immunoblot analysis of Foxo1 protein levels in Foxo1 vector- vs. control vectortreated mice using $\beta$-tubulin as control, as shown at the bottom of $\mathbf{B}$. Values shown in the $y$ axes are normalized to mock-treated controls. ${ }^{*} P<0.05$ by ANOVA; ${ }^{*} P<0.001$ by ANOVA.

production was associated with increased HDL cholesterol levels (Figure 6F), which accounted for relatively higher plasma cholesterol levels in Foxo ${ }^{\text {S253A }}$ transgenic mice.

Hepatic Foxo1 and apoC-III expression in diabetic mice. To study the physiological significance of Foxo1-mediated regulation of hepatic apoC-III expression, we determined hepatic Foxo1 levels in correlation with apoC-III expression in livers of type 1 and type 2 diabetic mice. These studies involved the use of diabetic NOD and $d b / d b$ mice, and their respective nonobese nondiabetic (NON) and $d b /+$ control animals. NOD mice are the commonly used genetic model of type 1 diabetes with spontaneous onset of diabetes by 12 weeks of age. Here, 1 group of severely diabetic NOD mice (average blood glucose levels, $554 \pm 18 \mathrm{mg} / \mathrm{dl}, n=6)$ and 1 group of age-matched NON mice $(122 \pm 12 \mathrm{mg} / \mathrm{dl}, n=6)$ were used. In addition, 1 group of diabetic $d b / d b$ mice ( $>600 \mathrm{mg} / \mathrm{dl}, n=6)$ and 1 group of their heterozygous littermates $(93 \pm 8 \mathrm{mg} / \mathrm{dl}, n=6)$ were also used. Using real-time RT-PCR, we determined the hepatic expression levels of Foxo1 and apoC-III mRNA in livers of diabetic NOD and $d b / d b$ mice and their respective control animals. We detected in liver of both diabetic NOD and $d b / d b$ mice, compared with controls, a significant increase in hepatic Foxo 1 abundance, along with a concomitant elevation in hepatic apoC-III expression (Figure 7, A and B). Consistent with these observations, plasma apoC-III and TG levels were markedly elevated in $d b / d b$ mice compared with littermate controls (Figure 7, C and D).

To study the potential alteration in Foxo1 subcellular distribution as a result of insulin deficiency or insulin insufficiency, we examined the localization of Foxo 1 in livers of diabetic NOD and $d b / d b$ mice by immunohistochemistry using anti-Foxo1 antibody. As shown in Figure 8, Foxo1 was immunostained mainly in the cytoplasm of liver cells in nondiabetic mice. In contrast, positive immunostaining was detected predominantly in the nucleus in liver of diabetic NOD and $d b / d b$ mice. These results are consistent with our previous studies, in which we subjected protein extracts of the nuclear and cytoplasmic fractions of hepatocytes isolated from diabetic $d b / d b$ and lean littermates to semi-quantitative immunoblot analysis (22). In that study, we found a quantitative (>3-fold) redistribution of Foxo1 from its cytoplasmic to nuclear location as the liver undergoes a shift from normal to insulin-resistant states (22).

Intestinal Foxo 1 and apoC-III expression. In addition to its hepatic expression, apoC-III is expressed in intestine. However, little is known about its regulation in response to insulin. Given the fact that intestine is an insulin- sensitive organ, intestinal apoC-III might be governed by insulin in a Foxo1-dependent mechanism. To test this hypothesis, we delivered Foxo 1 cDNA to the human intestinal Caco-2 cells and determined the level of apoC-III mRNA expression in the absence and presence of insulin at different concentrations in culture media. As shown in Figure 9A, adenovirus-mediated Foxo1 production significantly increased intestinal apoC-III expression in the absence of insulin. In the 

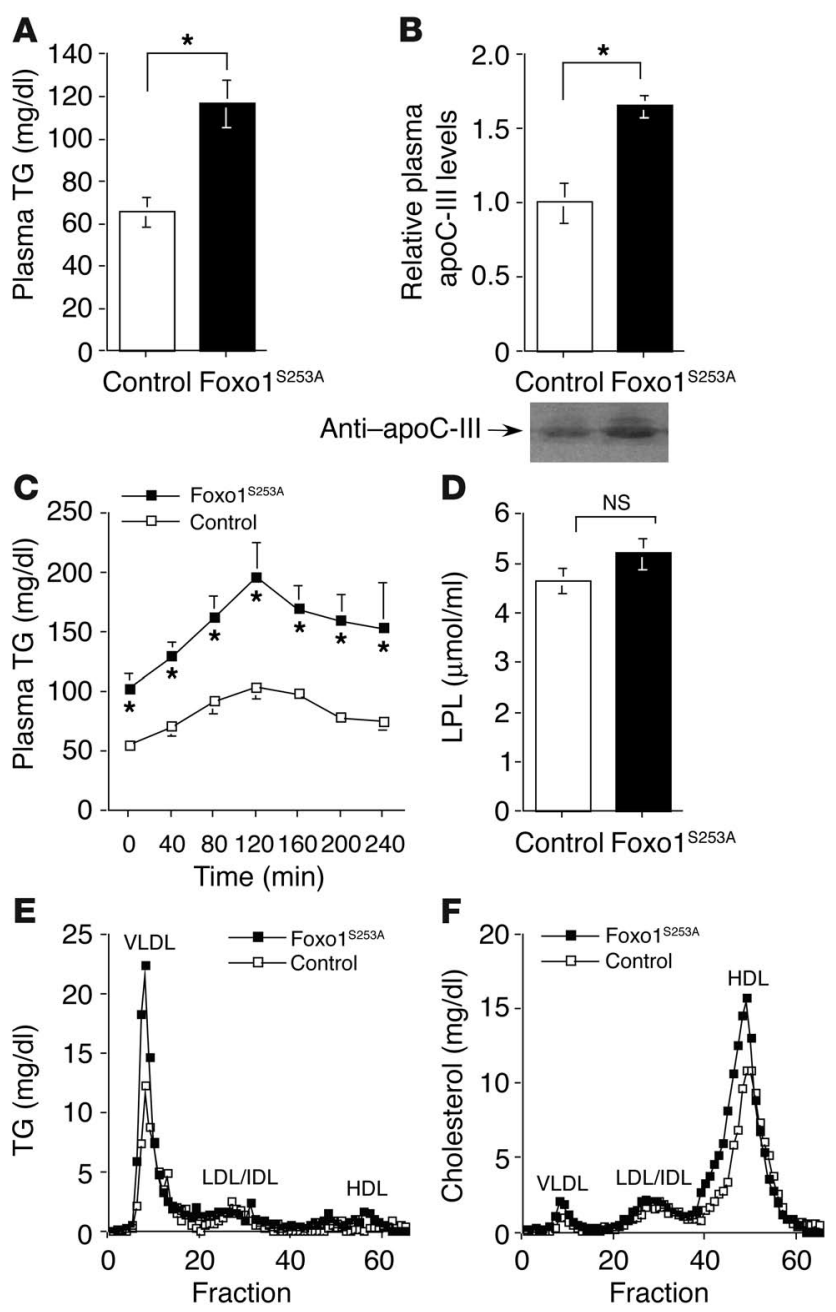

presence of insulin, Foxo1-mediated stimulation of apoC-III expression in Caco-2 cells was suppressed in an insulin concentration-dependent manner.

To corroborate these results, we examined the potential interaction between Foxo 1 protein and APOC 3 promoter in Caco- 2 cells. Caco- 2 cells were transduced by Foxo1 vector. After 24 hours of transduction, cells were subjected to ChIP analysis using anti-Foxo1 antibody or control IgG, or they were mock-treated identically following the ChIP protocol. As shown in Figure 9B, Foxo1 protein was detected in the products that were immunoprecipitated by anti-Foxo1 antibody, but not in control IgG- or mock-immunoprecipitated products. When analyzed by PCR, a specific DNA fragment (675 bp) corresponding to the nucleotide region $(-675 /+1)$ of the APOC3 promoter was amplified from the DNA products that were coimmunoprecipitated

\section{Figure 7}

Foxo1 and apoC-III expression in livers of NOD and $d b / d b$ mice. Diabetic NOD (18-week-old) and $d b / d b$ (6-month-old) mice, together with their respective NON and $d b /+$ controls, were killed. Foxo1 (A) and apoC-III (B) mRNA in liver were determined by real-time RT-PCR using $\beta$-actin mRNA as control. The relative levels of plasma TG (C) and apoC-III (D) were determined in diabetic and control mice. Data in $\mathbf{A}, \mathbf{B}$, and $\mathbf{D}$ are plotted as relative values after normalization to controls. ${ }^{*} P<0.05$ by ANOVA $(n=6) ;{ }^{* *} P<0.001$ by ANOVA.

\section{Figure 6}

Plasma TG metabolism in Foxo1 transgenic mice (4 months old). Foxo1 ${ }^{\mathrm{S} 253 \mathrm{~A}}$ transgenic mice $(n=8)$ and control littermates $(n=8)$ were studied for fasting plasma TG levels (A), plasma apoC-III levels (B), plasma TG profiles in response to fat tolerance test (C), and plasma LPL activity (D). Sera (500 $\mu$ l) pooled from individual mice were fractionated by fast-performance liquid chromatography through 2 consecutive Tricorn High-Performance Superose S-6 10/300GL Columns and 70 fractions ( $400 \mu \mathrm{l}$ per fraction) were collected and assayed for TG (E) and cholesterol levels (F). ${ }^{*} P<0.01$ by ANOVA.

by anti-Foxo1 antibody (Figure 9C). In contrast, no specific DNA was produced from control IgG or mock-immunoprecipitated products in the same PCR assay. Thus, similar to its action in hepatocytes, Foxo1 appeared to associate with the APOC 3 promoter in stimulating apoC-III expression in enterocytes.

To address the physiological significance of these findings, we studied intestinal Foxo1 production in correlation with apoC-III expression in intestine. Total RNA from intestine and liver of normal C56BL/6J mice (12 weeks old) was prepared and subjected to RT-PCR analysis. As shown in Figure 9D, Foxo1 was expressed in both liver and intestine, but its intestinal expression level in intestine was significantly lower than it was in liver. Likewise, a similar expression pattern was detected for apoC-III expression, with the liver being a major source of apoC-III production, which was consistent with the data in the literature (30).

To study the alterations in intestinal apoC-III expression in response to insulin deficiency or insulin resistance, we determined the expression level of apoC-III mRNA relative to that of $\beta$-actin mRNA in intestine of diabetic NOD and $d b / d b$ mice and then compared it to that in NON and $d b /+$ controls, respectively. As shown in Figure 9E, the relative apoC-III mRNA abundance in intestine of both NOD and $d b / d b$ mice was significantly increased, in comparison with nondiabetic controls. To study whether Foxo1 contributes
A

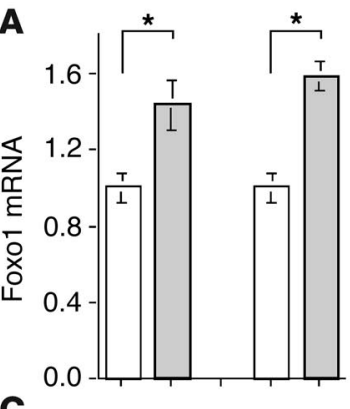

$$
\text { C }
$$

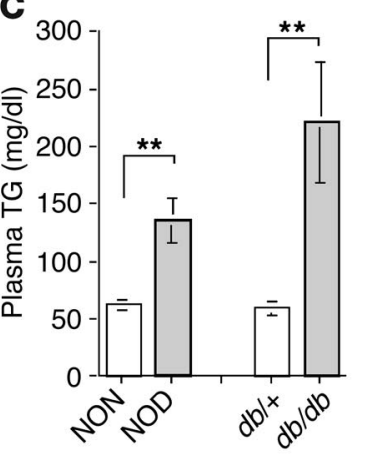

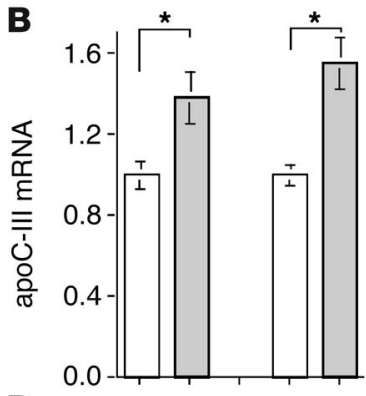

D

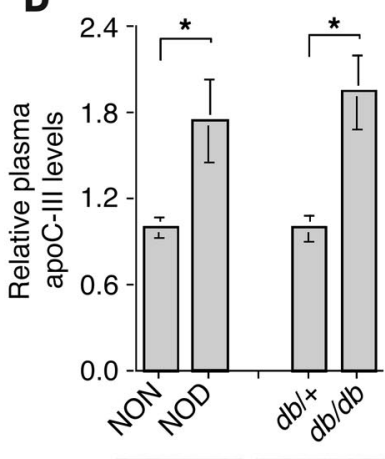

Anti-apoC-III 

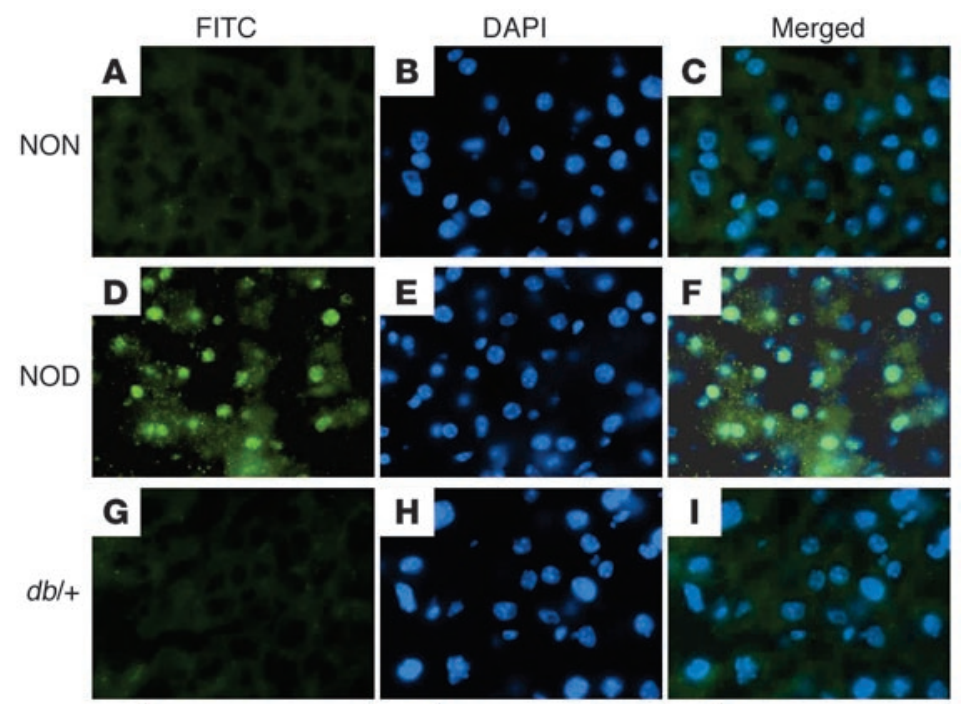

$d b / d b$
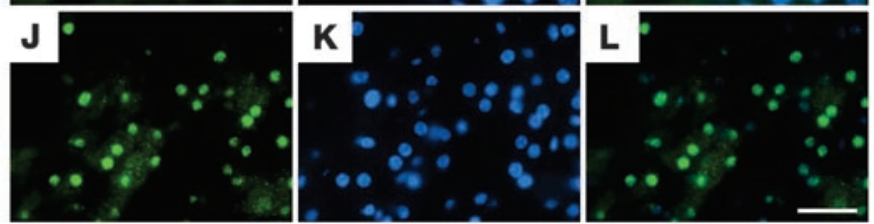

Figure 8

Immunohistochemistry. Liver tissues of diabetic NOD, $d b / d b$, and control mice were used for immunofluorescent labeling with rabbit anti-Foxo1 antibody (1:400 dilution). Foxo1 was immunostained green using donkey anti-rabbit IgG conjugated with FITC (1:200 dilution) (A, D, G, and J). Nuclei of hepatocytes were stained blue with DAPI (B, E, H, and $\mathbf{K})$. Merged images are shown in $\mathbf{C}$, F, I, and L. Scale bar: $200 \mu \mathrm{m}$.

to elevated apoC-III expression in intestine of diabetic NOD and $d b / d b$ mice, we determined intestinal Foxo1 mRNA abundance relative to that of $\beta$-actin mRNA. In comparison with nondiabetic controls, intestinal Foxo1 expression was significantly increased in insulin-deficient and insulin-resistant animals (Figure 9F). These results indicate that insulin deficiency and insulin insufficiency are invariably associated with elevated Foxo1 production in intestine, which might act as a contributing factor for increased intestinal apoC-III expression in diabetic NOD and $d b / d b$ mice.

\section{Discussion}

We have characterized the molecular mechanism underlying the inhibitory effect of insulin on apoC-III production. We have shown that in cultured hepatocytes, Foxo1 stimulated hepatic apoC-III expression, an effect that was counteracted by insulin and correlated with the characteristic property of Foxo1 to undergo insulin-dependent phosphorylation and nuclear exclusion $(16,31)$. Foxo1-ADA also stimulated hepatic apoC-III expression, but its stimulatory effect was indifferent to insulin inhibition, coinciding with the inability of insulin to induce Foxo1-ADA phosphorylation $(17,29)$. These results suggest that Foxo1, known as a mediator of insulin in governing gluconeogenesis in liver $(24,29)$, also functionally modulates hepatic apoC-III production in response to insulin.

Consistent with this interpretation, we show that a sequence $(-498 /-403)$ in the APOC3 promoter was able to associate with Foxo1. This region contains a consensus IRE, which has been implicated in mediating the inhibitory effect of insulin on apoC-III pro- duction (13). But to our knowledge, this putative IRE has not been shown to correlate with the ability of the APOC3 promoter to confer insulin responsiveness onto hepatic apoC-III expression. We show that deletion or site-directed mutagenesis of this IRE sequence rendered the mutant APOC3 promoter unresponsive to Foxo1 induction and insulin inhibition. These data provide evidence that the APOC3 promoter is a Foxo1 target, suggesting that this IRE regulates $A P O C 3$ promoter activity in response to insulin.

In keeping with the effect of Foxo1 on apoC-III expression in vitro, elevated Foxo 1 production in liver was shown to enhance hepatic apoC-III expression and perturb plasma TG metabolism in mice. Mice with elevated hepatic Foxo1 production exhibited relatively higher fasting plasma VLDL-TG levels, accompanied by significantly impaired fat tolerance. Furthermore, Foxo $1^{\mathrm{S} 253 \mathrm{~A}}$ transgenic mice developed hypertriglyceridemia, as manifested by their significantly elevated plasma apoC-III and VLDL-TG levels and impaired fat tolerance. These physiological data provide in vivo evidence that Foxo1 exerts a profound effect on plasma TG metabolism. A gain-of-function of Foxo1, caused by adenovirus-mediated hepatic production of Foxo 1 or transgenic expression of its constitutively active mutant in liver, is associated with enhanced hepatic apoC-III production and abnormal plasma TG metabolism in mice.

In addition to its hepatic production, apoC-III is produced in intestine at a relatively lower level (30). However, considerably less is known about the regulation of intestinal apoC-III expression in response to insulin. Here we demonstrate in cultured enterocytes that Foxo1 stimulates intestinal apoC-III expression. Its stimulatory effect was inhibited by insulin in a dose-dependent manner, which correlated with the ability of Foxo 1 to bind to the intestinal APOC3 promoter in Caco-2 cells. Thus, similarly to its hepatic expression of apoC-III, intestinal apoC-III production is negatively regulated by insulin via a Foxo1-dependent mechanism.

What is the physiological significance of Foxo1-mediated regulation of apoC-III expression? While apoC-III production is markedly elevated, accounting for impaired TG metabolism in poorly controlled subjects with both type 1 and type 2 diabetes $(12,32)$, the underlying pathophysiology is different. The former is due to the lack of insulin action to phosphorylate Foxo1, whereas the latter is caused by impaired insulin action to phosphorylate Foxo1 due to insulin resistance. Thus, despite the apparent difference in etiology, type 1 and type 2 diabetes share a common pathogenic feature of Foxo 1 deregulation. Consistent with this interpretation, we show that the production of Foxo1 was significantly elevated, accompanied by its increased nuclear localization in hepatocytes in insulin- deficient and insulin-resistant mice. Likewise, Foxo1 expression in intestine also became deregulated, which correlated with increased intestinal apoC-III production in diabetic NOD and $d b / d b$ mice. Our data are consistent with the notion that loss of insulin responsiveness in hepatic apoC-III expression is associated with hepatic apoC-III overproduction, which underlies the close relationship between insulin deficiency or resistance and the development of hypertriglyceridemia $(13,33)$.

Recently, Imae et al. (34) reported that hepatic Foxo1 expression and nuclear distribution were significantly elevated in liver in normal rats in response to prolonged fasting. Thus, in addition to its regulation at the level of transcription, the trans-activa- 


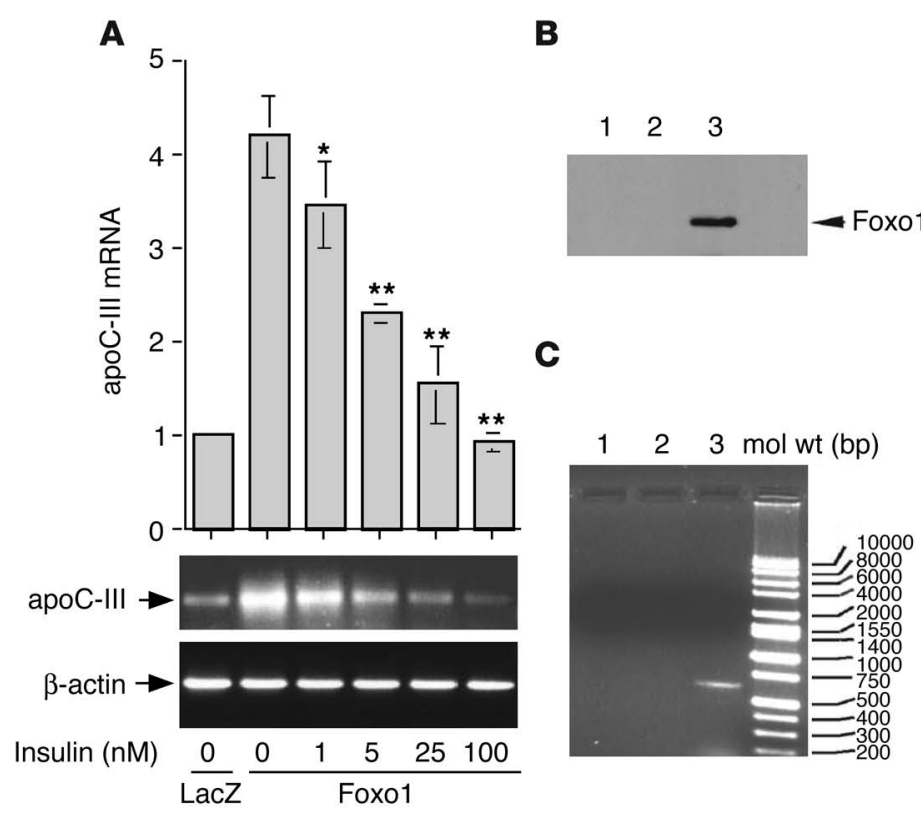

D

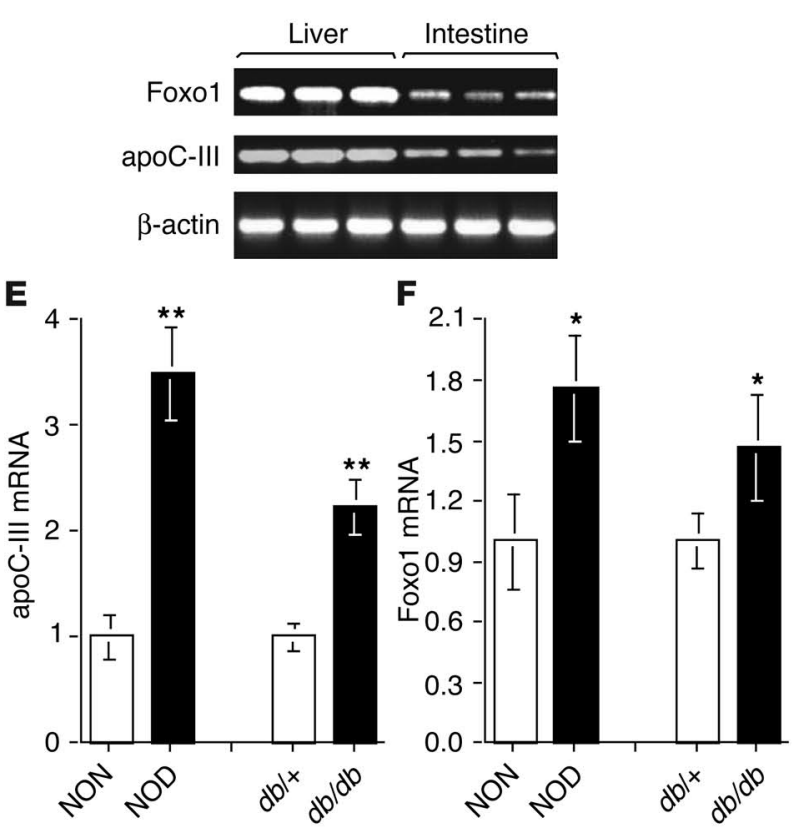

Figure 9

Effects of Foxo1 on intestinal apoC-III expression. (A) Foxo1-dependent regulation of apoC-III expression in Caco-2 cells. Cells were transduced with Foxo1 vector (MOI, 500 PFU/cell) in the absence and presence of insulin at indicated concentrations. After 24 hours, cells were harvested for the determination of endogenous apoC-III mRNA expression by real- time RT-PCR. The products of real-time RT-PCR were analyzed on $0.7 \%$ agarose gels and visualized under UV lights after ethidium bromide staining (below A). (B) Immunoblot. Foxo1 vector-transduced Caco-2 cells were subjected to ChIP analysis using anti-Foxo1 antibody, control sheep IgG, or PBS. Immunoprecipitates were studied by immunoblot analysis. (C) PCR analysis of coimmunoprecipitated DNA by ChIP. (D) Foxo1 and apoC-III expression in liver versus intestine. RNA (1 $\mu$ g) isolated from liver or intestine of lean C57BL/6J mice $(n=3)$ was analyzed by RT-PCR for Foxo1, apoC-III, and $\beta$-actin mRNA abundance. RT-PCR products were resolved on a $0.7 \%$ agarose gel and visualized under UV -light after staining with ethidium bromide. (E) Intestinal apoC-III mRNA levels. (F) Intestinal Foxo1 mRNA levels. The relative levels of apoC-III and Foxo1 mRNA in intestine of diabetic NOD and $d b / d b$ versus nondiabetic control mice were determined by real-time RT-PCR using $\beta$-actin mRNA as control. ${ }^{*} P<0.05$ and ${ }^{* *} P<0.001$ vs. controls.

tion activity of Foxo 1 is regulated at the post-translational level by altering its subcellular redistribution under physiological and pathophysiological conditions. A significant increase in Foxo1 production, accompanied by its skewed subcellular distribution in the nucleus, accounts for its enhanced trans-activation activity in stimulating hepatic and intestinal apoC-III production, resulting in impaired plasma TG metabolism in type 1 and type 2 diabetes. This raises the possibility that Foxo 1 acts as a molecular link between the loss of insulin inhibition of apoC-III production and the development of hypertriglyceridemia in diabetes.

In summary, we have shown that Foxo1 is an effective mediator of insulin signaling in modulating both hepatic and intestinal apoC-III expression. Under insulin-deficient and insulin-resistant conditions, Foxo 1 expression in liver and intestine becomes deregulated, contributing to the increased apoC-III production and impaired plasma TG metabolism associated with diabetes. Selective inhibition of Foxo1 function is predicted to inhibit its transcriptional activity and suppress unrestrained apoC-III production in liver, which will ameliorate diabetic dyslipidemia.

\section{Methods}

Cell culture and adenovirus-mediated transduction. HepG2 and Caco-2 cells were purchased from American Type Culture Collection (ATCC) and rat primary hepatocytes were obtained from Cambrex Corp. Cells were cultured in Vita- cell culture media (ATCC) as described (22). To transduce cells, we added to the cell culture $100 \mu \mathrm{l}$ of adenoviral vectors at a defined multiplicity of infection (MOI). After 24 hours of incubation, cells were collected for analysis. The adenoviral vectors used were as follows; Ad-CMV-Foxo 1 expressing wild-type Foxo1 (titer, $1.2 \times 10^{11} \mathrm{PFU} / \mathrm{ml}$ ), Ad-CMV-Foxo1-ADA expressing a constitutively active Foxo1 allele $\left(8.8 \times 10^{10} \mathrm{PFU} / \mathrm{ml}\right)$, Ad-CMV-Foxo1- $\Delta 256$ expressing a dominant-negative Foxo1 mutant $\left(1.6 \times 10^{11} \mathrm{PFU} / \mathrm{ml}\right)$, and Ad-RSV-LacZ expressing the control LacZ gene $\left(1.9 \times 10^{11} \mathrm{PFU} / \mathrm{ml}\right)$. All adenoviral vectors were produced in HEK293 cells and purified as described (22).

$R N A$ isolation and real-time RT-PCR. RNA preparation from cells or tissue and real-time RT-PCR for quantifying mRNA concentrations have been described (22). The primers for human APOC3 are 5'-ATGCACTGAGCAGCG-3' (hAPOC3 cDNA 134-148 nt) and 5'-ACGGCTGAAGTTGGT-3' (hAPOC3 cDNA, 276-290 nt). Mouse apoC-III primers are 5'-CAGCCCCGGACGCTCCTCAC3' (apoc3 cDNA, 4-23 nt) and 5'-CGACTCAATAGCTGGAGTTG-3' (apoc3 cDNA, 278-297 nt). Mouse apoa1 primers are 5'-GCTTGGCACGTATGGCAGCA-3' (apoa1 cDNA, 52-71 nt) and 5'-TCTCCAGGTTATCCCAGAAG-3' (apoa1 cDNA, 279-298 nt). Mouse apoA-IV primers are 5'-TTCCTGAAGGCTGCGGTGCT-3' (apoa4 cDNA, 4-23 nt) and 5'-CAGCTGTACGACAAAGGGCA-3' (apoa4 cDNA, 239-258 nt). Primers for real-time RT-PCR for Foxo1, GK, and $\beta$-actin mRNA have been described (22). All primers were obtained commercially from Integrated DNA Technologies.

EMSA. Electrophoretic mobility shift assay (EMSA) was used to study Foxo1 and DNA interaction. Foxo1 protein was prepared from a linked 
in vitro transcription-translation system using pHD334 containing Foxo1 cDNA driven by T7 RNA polymerase and using a linked transcription:translation kit (Ambion Inc.). After verifying the production of Foxo 1 by immunoblot analysis using anti-Foxo1 antibody, aliquots $(5 \mu \mathrm{g})$ of the translation products were mixed with radioisotope-labeled DNA probes in $10 \mathrm{mM}$ Tris- $\mathrm{Cl}$ ( $\mathrm{pH}$ 7.6), $10 \mathrm{mM} \mathrm{MgCl}_{2}, 100 \mathrm{mM} \mathrm{NaCl}$, and $1 \mathrm{mM}$ dithiothreitol, and then incubated at $30^{\circ} \mathrm{C}$ for 20 minutes to allow DNA-protein interaction. The samples were applied onto a nondenaturing polyacrylamide gel. After electrophoresis in $0.5 \times$ tris-borateEDTA buffer at $200 \mathrm{~V}$ for 30 minutes, the gel was dried in preparation for autoradiography. The probe was derived from a 27-bp DNA covering the putative IRE sequence of the human APOC3 promoter $(-467 /-440)$ $(33,35)$, which was radioactively labeled with $\left[\gamma^{-32} \mathrm{P}\right]$-ATP by $\mathrm{T} 4$ polynucleotide kinase (Amersham Biosciences). Likewise, a mutant probe was made of the same DNA except for 2 base substitutions, $-\mathrm{A} 458 \mathrm{C}$ and -A460G, in the IRE consensus sequence. As a positive control, a 26-bp synthetic DNA covering the IRE of the PEPCK promoter (-423/-398) (23) was radioactively labeled.

ChIP assay. Chromatin immunoprecipitation (ChIP) was used to study the interaction between Foxo1 and APOC3 promoter DNA in cells. HepG2 cells $\left(1 \times 10^{6}\right.$ cells) were transduced with Foxo 1 vector at an MOI of $50 \mathrm{PFU} / \mathrm{cell}$ in culture media. After 24 hours of incubation, cells were cross-linked with $1 \%$ formaldehyde, followed by sonication in a Microson 100-WW Ultrasonicator (Structure Probe Inc.) at 30\% of maximum power for 10-12 consecutive cycles of 10 -second pulses. After centrifugation at $18,000 \mathrm{~g}$ for 10 minutes, the supernatant was incubated with $5 \mu \mathrm{g}$ of anti-Foxo1 antibody, followed by immunoprecipitation using the ChIP kit (Upstate Biotechnology). As controls, aliquots $\left(1 \times 10^{6}\right)$ of Foxo 1 vector-transduced HepG2 cells were treated identically for the preparation of cell lysates, which were immunoprecipitated with $5 \mu \mathrm{g}$ of control IgG (sheep IgG, Rockland Immunochemicals) or PBS buffer. The immunoprecipitates were analyzed by immunoblot analysis for Foxo 1 and PCR for coimmunoprecipitated DNA.

Construction of the APOC3 promoter-directed luciferase reporter system. A 1.4-kb DNA fragment containing the human $A P O C 3$ promoter was amplified from human genomic DNA (BD Bioscience) by PCR using the primers for forward reaction (5'-GAATTCTGAGGGCAGAGCGG-3' [-1411/ $-1392 \mathrm{nt}]$ ) and for reverse reaction (5'-CTGCCTCTAGGGATGAACTG-3' $[5 / 24 \mathrm{nt}])$. After verifying its nucleotide sequence, the APOC3 promoter was cloned into a luciferase reporter plasmid pGL3 (Promega Corp.). To generate promoter variants, DNA fragments covering different lengths of the APOC 3 promoter were amplified by PCR using primers corresponding to the nucleotide sequence $(20 \mathrm{nt})$ at which the promoter was truncated and cloned into PGL3. In addition, PCR-based site-directed mutagenesis was used to create mutations at -458 and -460 , using a DNA primer corresponding to nucleotide sequence $-498 /-450$ with 2 substitutions, of $-\mathrm{A} 458 \mathrm{C}$ and $-\mathrm{A} 460 \mathrm{G}$, in the APOC 3 promoter.

Plasmid transfection and luciferase assay. HepG2 cells in 6-well microplates were transfected with $1 \mu \mathrm{g}$ of plasmid encoding the luciferase reporter gene under the control of different $A P O C 3$ promoter variants using the Lipofectamine 2000 (Invitrogen Corp.). In each transfection, $1 \mu \mathrm{g}$ of plasmid pCMV-LacZ was included and the amount of $\beta$-gal activity was used as a control to normalize transfection efficiency. After 24 hours of incubation, cells were collected and resuspended in $100 \mu \mathrm{l}$ of lysis buffer (Promega Corp.) for preparation of cell lysates. After centrifugation at $18,000 \mathrm{~g}$ for 2 minutes, aliquots $(20 \mu \mathrm{l})$ of the supernatant were used to determine luciferase activity using the Promega Luciferase Assay as well as $\beta$-gal activity using a $\beta$-gal reporter activity detection kit (SigmaAldrich). After normalizing to the amount of $\beta$-gal activity, the relative luciferase activity for each plasmid was reported.
Animal studies. Animals including C57BL/6J, CD-1, NOD, $d b / d b$, and littermate control mice at 6 weeks of age were purchased from the Jackson Laboratory. Foxo1 ${ }^{\mathrm{S} 253 \mathrm{~A}}$ transgenic mice and control littermates in a C57BL/6J genetic background were obtained from Domenico Accili's group (Columbia University). Animals were fed standard rodent chow and water ad libitum in sterile cages in a barrier animal facility with a 12-hour light/dark cycle. For vector administration, CD-1 mice were injected through tail veins with an adenoviral vector at the dose of $1.5 \times 10^{11} \mathrm{PFU} / \mathrm{kg}$ body weight, as described (22). After one week of treatment, animals were sacrificed by $\mathrm{CO}_{2}$ inhalation and liver tissues were frozen in liquid $\mathrm{N}_{2}$. Blood was collected from the tail vein into potassium-EDTA coated capillary tubes (Sarstedt) for plasma preparation, as previously described (22). Plasma levels of TG and cholesterol were determined using ThermoDMA Infinity TG and cholesterol reagents (Thermo Electron Corp.). All animal studies procedures were approved by the Institutional Animal Care and Usage Committee at the Mount Sinai School of Medicine (Protocol 01-0147).

Fat tolerance test. Mice were fasted overnight, followed by an oral bolus of olive oil at the dose of $20 \mu \mathrm{l} / \mathrm{g}$ body weight. Aliquots of tail vein blood $(20 \mu \mathrm{l})$ were sampled before and at different times after olive oil administration for determination of plasma TG levels.

Lipoprotein lipase assay. Mice were injected intravenously with 300 IU hepa$\mathrm{rin} / \mathrm{kg}$ body weight and tail-vein blood $(20 \mu \mathrm{l})$ was sampled 10 minutes after heparin infusion. Heparinized sera were prepared for the determination of lipoprotein lipase (LPL) activity using the LPL activity kit (Roar Biochemical, Inc.). This assay includes a nonfluorescent substrate emulsion that becomes intensely fluorescent upon interaction with LPL. Heparinized sera were diluted 10 -fold and $10 \mu \mathrm{l}$ of the diluted sera were mixed in a 96-well microplate with $200 \mu \mathrm{l}$ of substrate emulsion provided by the kit. After incubation at room temperature for 15 minutes, the reaction mixture was measured at $370-\mathrm{nm}$ excitation/450-nm emission for the determination of fluorescent intensity in a fluorescence microplate reader (Molecular Devices). In addition, a prehydrolyzed substrate (Roar Biochemical Inc.) at concentrations ranging from 2 to $30 \mu \mathrm{mol} / \mathrm{ml}$ was used for the construction of a standard curve, from which the relative amounts of LPL activity in sera were calculated.

Semi-quantitative immunoblot assay. A semi-quantitative immunoblot assay was performed to determine plasma apoC-III protein levels. Aliquots of plasma (20 $\mu \mathrm{g}$ plasma proteins) were resolved on $15 \%$ SDSpolyacrylamide gels. Proteins were blotted onto a piece of nitrocellulose membrane, and then probed with goat anti-apoC-III antibody (1:2,000 dilution, Abcam Inc.), followed by incubation with anti-goat IgG conjugated with horseradish peroxidase (HRP) (1:1,000 dilution). Proteins were detected by the ECL detection reagents (Amersham Biosciences) and their relative intensities were quantified by densitometry using the NIH Image software as described (22). For detection of Foxo1, hepatic protein extracts $(20 \mu \mathrm{g})$ were resolved on $10 \%$ polyacrylamide gels, followed by immunoblot analysis using rabbit anti-Foxo1 antibody (1:4,000, Cell Signaling Biotechnology) or monoclonal anti- $\beta$-tubulin antibody $(1: 1,000$, Sigma-Aldrich) as control as described (22).

Fractionation of lipoproteins by fast-performance liquid chromatography. Aliquots $(400 \mu \mathrm{l})$ of plasma pooled from Foxo1 vector-transfected, LacZ vector-transfected, or mock-treated mice ( $n=6$ per group) were applied to the Tricorn High-Performance Superose S-6 10/300GL Column using a fast-performance liquid chromatography system (Amersham Biosciences), followed by elution with PBS at a constant flow rate of $0.2 \mathrm{ml} / \mathrm{min}$. Fractions $(200 \mu \mathrm{l})$ were eluted and assayed for triglyceride and cholesterol concentrations using the ThermoDMA Infinity TG and cholesterol reagents (Thermo Electron Corp.).

Immunohistochemistry. Animals were killed by $\mathrm{CO}_{2}$ inhalation and liver tissue was embedded with Histoprep tissue embedding media (Fisher 
Scientific). Frozen tissue was cut into thin sections $(8 \mu \mathrm{m})$ and subjected to immunohistochemistry using rabbit anti-Foxo1 (dilution at 1:400, Cell Signaling Biotechnology), as described (36). The secondary antibody was anti-rabbit IgG conjugated with fluorescein (FITC, Jackson ImmunoResearch Laboratories). The nuclei of hepatocytes were visualized by immunostaining with DAPI (Sigma-Aldrich).

Statistics. Statistical analyses of data were performed by analysis of variance (by ANOVA) using StatView software (Abacus Concepts, Inc.). Pairwise comparisons were performed to study the significance between different conditions. Data were expressed as mean \pm SE. $P$ values less than 0.05 were considered significant.

\section{Acknowledgments}

This work was supported by NIH grant DK59892. We thank Zachary Bloomgarden, Alex Ma, and Charles Mobbs for critical reading of this manuscript. We would like to thank Domenico
Accili for providing Foxo $1^{\mathrm{S} 253 \mathrm{~A}}$ transgenic mice and for critical advice for this study.

Received for publication September 8, 2003, and accepted in revised form September 14, 2004.

Address correspondence to: Hengjian Henry Dong, Department of Pediatrics, Rangos Research Center, Children's Hospital of Pittsburgh, University of Pittsburgh School of Medicine, Pittsburgh, Pennsylvania 15213, USA. Phone: (412) 692-6577; Fax: (412)692-5809; Email: dongh@pitt.edu.

Hengjian Henry Dong's present address is: Department of Pediatrics, Rangos Research Center, Children's Hospital of Pittsburgh, University of Pittsburgh School of Medicine, Pittsburgh, Pennsylvania, USA.
1. Wang, C., McConathy, W.J., Kloer, H.J., and Alaupovic, P. 1985. Modulation of lipoprotein lipase activity by apolipoproteins: effect of apolipoprotein C-III. J. Clin. Invest. 75:384-390.

2. McConathy, W.J., Gesquiere, J.C., Bass, H., Tartar, A., and Fruchart, J.C. 1992. Inhibition of lipoprotein lipase activity by synthetic peptides of apolipoprotein C-III. J. Lipid Res. 33:995-1003.

3. Kinnunen, P.K.J., and Ehnholm, C. 1976. Effect of serum and $\mathrm{C}$ apolipoproteins from very low density lipoproteins on human post-heparin plasma hepatic lipase. FEBS Lett. 65:354-357.

4. Wuarfordt, S.H., Michalopoulos, G., and Schirmer, B. 1982. The effect of human C apolipoproteins on the in vitro hepatic metabolism of triglyceride emulsions in the rat. J. Biol. Chem. 257:14642-14647.

5. Windler, E., and Havel, R.J. 1985. Inhibitory effect of $\mathrm{C}$ apolipoprotein from rats and humans on the uptake of triglyceride-rich lipoproteins and their remnants by the perfused rat liver. J. Lipid Res. 26:556-565.

6. Mann, C.J., et al. 1997. Inhibitory effects of specific apolipoprotein C-III isoforms on the binding of triglyceride-rich lipoproteins to the lipolysis-stimulated receptor. J. Biol. Chem. 272:31348-31354.

7. Ito, Y.N., Azrolan, A., O'Connell, A., Walsh, A., and Breslow, J.L. 1990. Hypertriglyceridemia as a result of human apo CIII gene expression in transgenic mice. Science. 249:790-793.

8. Shachter, N.S. 2001. Apolipoproteins C-1 and C-III as important modulators of lipoprotein metabolism. Curr. Opin. Lipidol. 12:297-304.

9. Maeda, N., et al. 1994. Targeted disruption of the apolipoprotein C-III gene in mice results in hypotriglyceridemia and protection from postprandial hypertriglyceridemia.J Biol. Chem. 269:23610-23616.

10. Hirano, T., et al. 2001. Apoprotein C-III deficiency markedly stimulates triglyceride secretion in vivo: comparison with apoprotein B. Amer. J. Physiol. 281:E665-E669.

11. Jong, M.C., et al. 2001. Apolipoprotein C-III deficiency accelerates triglyceride hydrolysis by lipoprotein lipase in wild-type and apoE knockout mice. J. Lipid Res. 42:1578-1585.

12. Chen, M., Breslow, J.L., Li, W., and Leff, T. 1994 Transcriptional regulation of the apoC-III gene by insulin in diabetic mice: correction with changes in plasma triglyceride levels. J. Lipid Res. 35:1918-1924.

13. Li, W.W., et al. 1995. Common genetic variation in the promoter of the human apo CIII gene abol- ishes regulation by insulin and may contribute to hypertriglyceridemia. J. Clin. Invest. 96:2601-2605.

14. Accili, D., and Arden, K.C. 2004. FoxOs at the crossroads of cellular metabolism, differentiation, and transformation. Cell. 117:421-426.

15. Kops, G.J., and Burgering, B.M. 1999. Forkhead transcription factors: new insights into protein kinase B (c-akt) signaling. J. Mol. Med. 77:656-665.

16. Nakae, J., Park, B.C., and Accili, D. 1999. Insulin stimulates phosphorylation of the forkhead transcription factor FKHR on serine 253 through a wortmannin-sensitive pathway. J. Biol. Chem. 274:15982-15985.

17. Nakae, J., Barr, V., and Accili, D. 2000. Differential regulation of gene expression by insulin and IGF-1 receptors correlates with phosphorylation of a single amino acid residue in the forkhead transcription factor FKHR. EMBO J. 19:989-996.

18. Durham, S.K., et al. 1999. FKHR binds the insulin response element in the insulin-like growth factor binding protein-1 promoter. Endocrinology. 140:3140-3146.

19. Biggs, W.H., III, Meisenhelder, J., Hunter, T., Cavenee, W.K., and Arden, K.C. 1999. Protein kinase B/ Akt-mediated phosphorylation promotes nuclear exclusion of the winged helix transcription factor FKHR1. Proc. Natl. Acad. Sci. U. S. A. 96:7421-7426.

20. Rena, G., Guo, S., Cichy, S.C., Unterman, T.G., and Cohen, P. 1999. Phosphorylation of the transcription factor forkhead family member FKHR by protein kinase B. J. Biol. Chem. 274:17179-17183.

21. Rena, G., et al. 2002. Two novel phosphorylation sites on FKHR that are critical for its nuclear exclusion. EMBO J. 21:2263-2271.

22. Altomonte, J., et al. 2003. Inhibition of Foxo1 function is associated with improved fasting glycemia in diabetic mice. Am. J. Physiol. 285:E718-E728.

23. O’Brien, R.M., Lucas, P.C., Forest, C.D., Magnuson, M.A., and Granner, D.K. 1990. Identification of a sequence in the PEPCK gene that mediates a negative effect of insulin on transcription. Science. 249:533-537.

24. Nakae, J., Kitamura, T., Silver, D.L., and Accili, D. 2001. The forkhead transcription factor Foxo1 (Fkhr) confers insulin sensitivity onto glucose-6 phosphatase expression. J. Clin. Invest. 108:1359-1367. doi:10.1172/JCI200112876.

25. Schmoll, D., et al. 2000. Regulation of glucose-6phosphatase gene expression by protein kinase $\mathrm{B}$ alpha and the forkhead transcription factor FKHR
Evidence for insulin response unit-dependent and -independent effects of insulin on promoter activity. J. Biol. Chem. 275:36324-36333.

26. Huard, J., et al. 1995. The route of administration is a major determinant of the transduction efficiency of rat tissues by adenoviral recombinants. Gene Ther. 2:107-115.

27. Bruns, G.A., Karathanasis, S.K., and Breslow, J.L. 1984. Human apolipoprotein A-I--C-III gene complex is located on chromosome 11. Arteriosclerosis. 4:97-102.

28. Stan, S., Delvin, E., Lambert, M., Seidman, E., and Levy, E. 2003. Apo A-IV: an update on regulation and physiologic functions. Biochim. Biophys. Acta. 1631:177-187.

29. Nakae, J., et al. 2002. Regulation of insulin action and pancreatic $\beta$-cell function by mutated alleles of the gene encoding forkhead transcription factor Foxo1. Nat. Genet. 32:245-253.

30. Haddad, I.A., Ordovas, J.M., Fitzpatrick, T., and Karathanasis, S.K. 1986. Linkage, evolution, and expression of the rat apolipoprotein A-I, C-III, and A-IV genes. J. Biol. Chem. 261:13268-13277.

31. Nakae, J., Kitamura, T., Ogawa, Y., Kasuga, M., and Accili, D. 2001. Insulin regulation of gene expression through the forkhead transcription factor Foxo1 (Fkhr) requires kinases distinct from Akt. Biochemistry. 40:11768-11776.

32. Gervaise, N., Garrigue, M.A., Lasfargues, G., and LeGomte, P. 2000. Triglycerides, apo C3 and LP $\mathrm{B}: \mathrm{C} 3$ and cardiovascular risk in type II diabetes. Diabetologia. 43:703-708.

33. Dallinga-Thie, G.M., Groenendijk, M., Blom, R.N., De Bruin, T.W., and De Kant, E. 2001. Genetic heterogeneity in the apolipoprotein C-III promoter and effects of insulin. J. Lipid Res. 42:1450-1456.

34. Imae, M., Fu, Z., Yoshida, A., Noguchi, T., and Kato, H. 2003. Nutritional and hormonal factors control the gene expression of FoxOs, the mammalian homologues of DAF-16. J. Mol. Endocrinol. 30:253-262.

35. Groenendijk, M., et al. 1999. Association of plasma lipids and apolipoproteins with the insulin response element in the apoC-III promoter region in familial combined hyperlipidemia. J. Lipid Res. 40:1036-1044.

36. Kitamura, T., et al. 2002. The forkhead transcription factor Foxo1 links insulin signaling to Pdx1 regulation of pancreatic beta cell growth. J. Clin. Invest. 110:1839-1847. doi:10.1172/JCI200216857. 